\title{
De novo KCNH1 mutations in four patients with syndromic developmental delay, hypotonia and seizures
}

\author{
Ryoko Fukai ${ }^{1,2}$, Hirotomo Saitsu ${ }^{1}$, Yoshinori Tsurusaki ${ }^{1}$, Yasunari Sakai ${ }^{3}$, Kazuhiro Haginoya ${ }^{4}$, \\ Kazumasa Takahashi ${ }^{5}$, Monika Weisz Hubshman ${ }^{6,7,8}$, Nobuhiko Okamoto ${ }^{9}$, Mitsuko Nakashima ${ }^{1}$, \\ Fumiaki Tanaka ${ }^{2}$, Noriko Miyake ${ }^{1}$ and Naomichi Matsumoto ${ }^{1}$
}

The voltage-gated Kv10.1 potassium channel, also known as ether-a-go-go-related gene 1, encoded by KCNH1 (potassium voltage-gated channel, subfamily $\mathrm{H}$ (eag related), member 1 ) is predominantly expressed in the central nervous system. Recently, de novo missense $K C N H 1$ mutations have been identified in six patients with Zimmermann-Laband syndrome and in four patients with Temple-Baraitser syndrome. These syndromes were historically considered distinct. Here we report three de novo missense $K C N H 1$ mutations in four patients with syndromic developmental delay and epilepsy. Two novel $K C N H 1$ mutations (p.R357Q and p.R357P), found in three patients, were located at the evolutionally highly conserved arginine in the channel voltage-sensor domain (S4). Another mutation (p.G496E) was found in the channel pore domain (S6) helix, which acts as a hinge in activation gating and mainly conducts non-inactivating outward potassium current. A previously reported p.G496R mutation was shown to produce no voltage-dependent outward current in CHO cells, suggesting that p.G496E may also disrupt the proper function of the $\mathrm{Kv}$ channel pore. Our report confirms that $K C N H 1$ mutations are associated with syndromic neurodevelopmental disorder, and also support the functional importance of the S4 domain.

Journal of Human Genetics (2016) 61, 381-387; doi:10.1038/jhg.2016.1; published online 28 January 2016

\section{INTRODUCTION}

In humans, at least 12 voltage-gated potassium channel (Kv) subfamilies (Kv1 to Kv12) contribute to neuronal signaling in the nervous system. ${ }^{1,2} \mathrm{KCNH}$ channels are important regulators of cellular excitability and have been associated with cancer, ${ }^{3}$ cardiac long QT syndrome type 2, epilepsy and schizophrenia. ${ }^{4}$ Heterozygous mutations in $\mathrm{Kv}$ are involved in epileptic disorders: KCNA1 (Kv1.1), KCNA2 (Kv1.2), KCNC1 (Kv3.1), KCNQ2 (Kv7.2), KCNQ3 (Kv7.3) and KCNH5 (Kv10.2).,5-12 The Kv10.1 channel encoded by KCNH1 belongs to the ether-a-go-go family within the Kv family. ${ }^{1} \mathrm{KCNH} 1$ is expressed in diverse regions of the central nervous system ${ }^{13}$ including the hippocampus, ${ }^{14}$ and consists of tetrameric $\alpha$-subunits, with each subunit containing six membrane-spanning $\alpha$-helices (S1-S6). ${ }^{15,16}$ Of these transmembrane $\alpha$-helices, S1-S4 segments act as voltagesensor domains, and S5 and S6 form a pore-lining loop. ${ }^{15,16}$ Although severe neuronal developmental impairment was observed in the zebrafish knockdown of $k c n h 1,{ }^{17}$ the role of $K C N H 1$ in human diseases is under investigation.
Previously, heterozygous KCNH1 (Kv10.1) mutations have been reported in six patients with Zimmermann-Laband syndrome (ZLS), and in four patients with Temple-Baraitser syndrome (TBS). ${ }^{10,15}$ These patients showed developmental delay, various types of infantile-onset seizures, some had dysmorphic faces, abnormal muscle tone, aplastic/hypoplastic thumb or toe nails and thick and broad toes, ${ }^{10,15}$ suggesting that $\mathrm{KCNH} 1$ mutations may cause a phenotypic continuum of neurodevelopmental disorders with some distinctive dysmorphic features. Functional analysis showed that all the de novo mutations, except for p.Gly496Arg causing outward rectifying currents, ${ }^{15}$ resulted in decreasing the activation threshold to the negative potentials. ${ }^{10,15}$ Therefore, mutations in KCNH1 show hyperactive effects may disrupt cell proliferation and neuronal activity. ${ }^{10,15}$

In this study, we identified three de novo heterozygous $\mathrm{KCNH} 1$ mutations in four different patients with syndromic developmental delay and infantile epilepsy. The nature of the mutations and the clinical features of the patients are described and discussed.

\footnotetext{
${ }^{1}$ Department of Human Genetics, Yokohama City University Graduate School of Medicine, Yokohama, Japan; ${ }^{2}$ Department of Neurology and Stroke Medicine, Yokohama City University Graduate School of Medicine, Yokohama, Japan; ${ }^{3}$ Department of Pediatrics, Graduate School of Medical Sciences, Kyushu University, Fukuoka, Japan; ${ }^{4}$ Department of Pediatric Neurology, Takuto Rehabilitation Center for Children, Sendai, Japan; ${ }^{5}$ Department of Pediatrics, Yamaguchi University Graduate School of Medicine, Ube, Japan; ${ }^{6}$ Pediatric Genetics Unit, Schneider Children's Medical Center of Israel, Petach Tikva, Israel; ${ }^{7}$ Raphael Recanati Genetic Institute, Rabin Medical Center, Petach Tikva, Israel; ${ }^{8}$ Sackler Faculty of Medicine, Tel Aviv University, Tel Aviv, Israel and ${ }^{9}$ Department of Medical Genetics, Osaka Medical Center and Research Institute for Maternal and Child Health, Izumi, Japan

Correspondence: Dr N Matsumoto, Department of Human Genetics, Yokohama City University Graduate School of Medicine, Fukuura 3-9, Kanazawa-ku, Yokohama 2360004, Japan.

E-mail: naomat@yokohama-cu.ac.jp

Received 10 September 2015; revised 26 November 2015; accepted 3 January 2016; published online 28 January 2016
} 


\section{MATERIALS AND METHODS}

\section{Case reports}

Patient 1. This 8-year-old boy was born, without asphyxia, to healthy, non-consanguineous parents after an uneventful pregnancy (40 gestational weeks). His birth weight, height and occipitofrontal circumference (OFC) were 3446 g (+0.6 s.d.), $49.0 \mathrm{~cm}(+0.5$ s.d.) and $35.0 \mathrm{~cm}$ (+1 s.d.), respectively. His first seizure, a generalized tonic-clonic seizure, occurred at 6 months of age. On examination, he had moderate developmental delay (developmental quotient: 50) and hypotonia with minor anomalies including a long philtrum and thick upper lip. He also had long eyelashes and broad thumbs of his hand and first toes of his feet. The electroencephalograph (EEG) showed spikes. Routine biochemical analyses were all normal and chromosomal G-banding was 46,XY (normal). Brain magnetic resonance imaging (MRI) at age 11 months showed no abnormality. He underwent physiotherapy and his idiopathic epilepsy was controlled with valproic acid and zonisamide. He achieved head control at 5 months, sitting alone at 1 year and self-supported standing at 3 years. An EEG at 1 year showed several small spikes in the right frontal and central areas. On examination at 7 years, he could sit alone, roll over and crawl, but speak no meaningful words. His body weight, height and OFC were $21.0 \mathrm{~kg}$ ( -0.9 s.d.), $121.8 \mathrm{~cm}$ ( -0.5 s.d.) and $49.5 \mathrm{~cm}$ ( -1.6 s.d.).

Patient 2. This 6-year-old boy was born without asphyxia to healthy parents after an uneventful pregnancy (40 gestational weeks). There was no family history of epilepsy, but his father was deaf. His birth weight was $4000 \mathrm{~g}$ (90-95 percentile). His first seizure was at 1 month, and the EEG showed temporal focus spikes. His seizures were controlled by combination therapy with frisium, trileptin and risperdal. On examination, he showed severe developmental delay and hypertonia. He had a coarse face with thick eyebrows, long eyelashes, epicanthic folds, a broad nasal bridge, full cheeks, a long philtrum, thick lips, an open mouth, gingival enlargement, and abnormal and delayed dentition (Figure 1; Table 1). Bilateral broad and long first toes and clinodactyly of his fifth toes are shown in Figure 1. Laboratory tests including urinary amino acids, blood lactate, pyruvate, thyroid stimulating hormone and routine biochemical analyses were all normal.

Patient 3. This 7-year-old boy was born without asphyxia to healthy, nonconsanguineous parents after an uneventful pregnancy (40 gestational weeks). There was no family history of epilepsy. His birth weight, length and OFC were $2872 \mathrm{~g}$ ( -0.3 s.d.), $48 \mathrm{~cm}$ ( -0.5 s.d.) and $33 \mathrm{~cm}$ ( -0.2 s.d.), respectively. His first seizure was at 4 months, when he had a generalized tonic-clonic seizure that lasted $2 \mathrm{~min}$. An EEG showed rare spikes in the right central area, and carbamazepine was started. Since then, he has had five focal seizures of his right upper extremities with secondary generalization. He was then referred to our hospital for evaluation of developmental delay and epilepsy at 9 months. On examination, he had moderate developmental delay (developmental quotient: 45) and hypotonia with minor anomalies including mild hypertelorism, bilateral ptosis, a broad nasal bridge, loss of nasolabial fold, anteverted nostrils, a long philtrum, an open mouth, thick lips, downturned corners of a triangular mouth and a right simian crease. He had broad toes, but no hypoplasia of his toenails. He had a pseudomyopathic face. Laboratory tests, including urinary amino acids, blood lactate, pyruvate, thyroid stimulating hormone and routine biochemical analyses, were all normal. Chromosomal G-banding was normal as was tandem mass screening of the urine. Brain MRI at 11 months was also normal. He was diagnosed with intellectual disability with idiopathic epilepsy, and treated with physiotherapy and carbamazepine. He achieved head control at 6 months, sitting alone at 13 months and standing at 2.5 years. An EEG at 2 years and 4 months showed several small spikes in the right central area.

On examination at 6 years, he could sit alone, roll over and crawl, but had no meaningful words. His developmental quotient was 13 , and his body weight, height and OFC were $15.4 \mathrm{~kg}$ ( -1.5 s.d.), $106.5 \mathrm{~cm}(-1.3$ s.d.) and $49.7 \mathrm{~cm}$ $(-1.1$ s.d.), respectively. He had pes planovalgus and hypotonia. He also had constipation, gingival hypertrophy and delayed dentition. He had mild choreoathetotic movement of his extremities, which was exaggerated with febrile illness. Brain MRI was normal, and an EEG showed disorganized waking background for his age, and high-voltage slow waves and equivocal spikes were noted in the bilateral frontal areas during sleep and wakefulness. He had eight seizures during the previous year, which were focal clonic seizures of his right arm and face, and automatic seizures of his right arm, which sometimes developed to generalized tonic-clonic convulsions. The seizure duration was always within $3 \mathrm{~min}$ without status epilepticus. He was treated with carbamazepine and clobazam, which showed relative effectiveness. Other anticonvulsants, including levetiracetam, topiramate, zonisamide, valproic acid and lamotrigine, were ineffective.

Patient 4. This 3-year-old boy was born without asphyxia, the third child to healthy non-consanguineous parents after a 38-week uneventful pregnancy. His two older sisters were healthy. His birth weight, length and OFC were $2334 \mathrm{~g}$ (-1.6 s.d.), $46.5 \mathrm{~cm}$ (-1.2 s.d.) and $32 \mathrm{~cm}$ (mean), respectively. He presented with generalized convulsions within $24 \mathrm{~h}$ of his birth, and was admitted to the neonatal care unit for 4 days. An EEG did not detect paroxysmal activity and there was no recurrence of seizures until he was 43 days old. He fed well with milk, but failed to gain body weight as expected during his first month.

On days 43 and 46 , he stopped breathing and moving for 7-10 s, which was accompanied by cyanosis and general tonic-clonic seizures lasting for $1-2 \mathrm{~min}$ for each episode. He was readmitted to the hospital on day 50 , presenting with continual hyperirritability, myoclonus and intermittent oculogyric movements. He was referred to our department at age 56 days. On admission, he was $55.5 \mathrm{~cm}$ in length $(-1.9$ s.d.), weighed $3.8 \mathrm{~kg}(-3.5$ s.d.) and had an OFC of $38.8 \mathrm{~cm}$ ( -0.7 s.d.). Interictal EEG recordings revealed sporadic, multifocal spikes and sharp waves. Brain MRI at 61 days was normal. He had multiple minor anomalies including large ears, a high-arched palate and bilateral ptosis (Table 1). Neurologically, muscular hypotonia was remarkable, whereas deep tendon reflexes were brisk. Routine biochemical analyses were all normal, and G-band karyotyping was 46, XY.

For epileptic seizures, valproic acid, clobazam and levetiracetam were effective in combination. Meaningful words have never been acquired. At age 12 months, he was $77.8 \mathrm{~cm}$ in height (+0.9 s.d.), he weighed $8.41 \mathrm{~kg}(-1.1 \mathrm{s.d}$.) and his $\mathrm{OFC}$ was $47.0 \mathrm{~cm}$ ( +0.5 s.d.). Brain MRI at 1 year 2 months showed no brain malformation but a moderately decreased brain volume was noticed. Photographs in Figure 1 were taken at 1 year and 11 months. At 3 years, his height and body weight are $11.6 \mathrm{~kg}(-1.3$ s.d.) and $96 \mathrm{~cm}(+1.1$ s.d.), respectively. He can not control his head or sit alone.

\section{Whole-exome sequencing}

Genomic DNA was extracted from the peripheral blood of patient and parents. Approximately, 3- $\mu$ g DNA was sheared and used for a SureSelect Human All Exon V4 or V5 library (Agilent Technologies, Santa Clara, CA) according to the manufacturer's instructions. Captured DNA was sequenced on an Illumina HiSeq2000 (Illumina, San Diego, CA) with 101-bp paired-end reads. Image analysis and base calling were performed by sequence control software real-time analysis and CASAVA software v1.8 (Illumina). The quality controlled (Path Filter) reads were mapped to the human reference genome (UCSC hg19, NCBI build 37), using Novoalign (http://www.novocraft.com/). After the removal of PCR duplication by Picard 1.55 (http://broadinstitute.github.io/picard/), singlenucleotide variants, and short insertions and deletions were identified using Genome Analysis Toolkit (http://www.broadinstitute.org/gatk/). These singlenucleotide variants, and insertions and deletion were annotated using ANNOVAR (http://www.openbioinformatics.org/annovar/). This allowed the removal of common variants registered in dbSNP137 (minor allele frequency $\geqslant 0.01$ ). All variants within exons or $\pm 30 \mathrm{bp}$ from exon-intron boundaries, those registered in dbSNP137, the National Heart, Lung and Blood Institute Exome Sequencing Project Exome Variant Server (NHLBI-ESP 6500, http://evs.gs. washington.edu/EVS/), or our in-house database (exome data from 575 Japanese individuals) were removed. Variants were confirmed by Sanger sequencing using an ABI PRISM 3500xl autosequencer (Life Technologies, Carlsbad, CA). In this analysis, mutations were annotated based on $\mathrm{KCNH} 1$ isoform1, NM_172362.2, NP_758872. This study was approved by the Institutional Review Board of Yokohama City University School of Medicine. Written informed consent was obtained from patients or parents. 

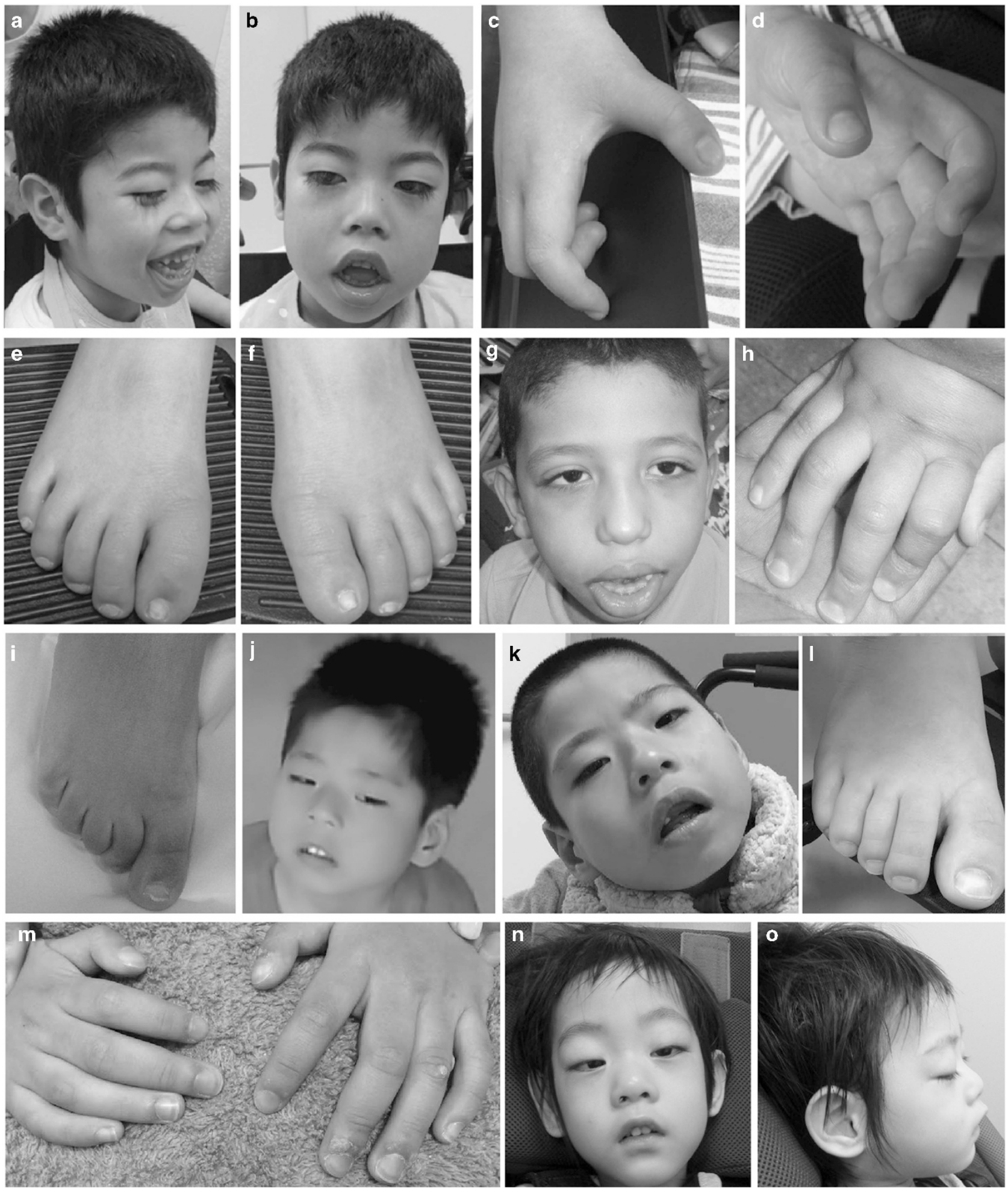

Figure 1 Clinical features of four patients with a $\mathrm{KCNH1}$ mutation. Facial appearance (a, b), right hand (c), left hand (d), right foot (e) and left foot (f) of patient 1. Long eyelashes, hypoplastic toe nails and broad thumbs and toes are evident. Facial appearance (g), right hand (h) and right foot (i) of patient 2. Hypoplastic toe nails and anonychia of the toes are apparent. Facial appearance at 4 years old (j) and 6 years old (k), right foot, showing broad first toe (I), right and left hands $(\mathbf{m})$ of patient 3. Facial appearance $(\mathbf{n}, \mathbf{o})$ of patient 4. A full color version of this figure is available at the Journal of Human Genetics journal online.

\section{RESULTS}

Identification of de novo KCNH1 mutations

We identified three novel missense $\mathrm{KCNH1}$ mutations by whole-exome sequencing (WES) in four unrelated patients with developmental delay and epilepsy. Trio-based WES was performed in two patients, and the other two were analyzed by proband-only WES. Each WES performance is shown in Supplementary Table 1. We focused on rare nonsynonymous variants that were absent in 
Table 1 Genetic and clinical features in patients with KCNH1 mutations

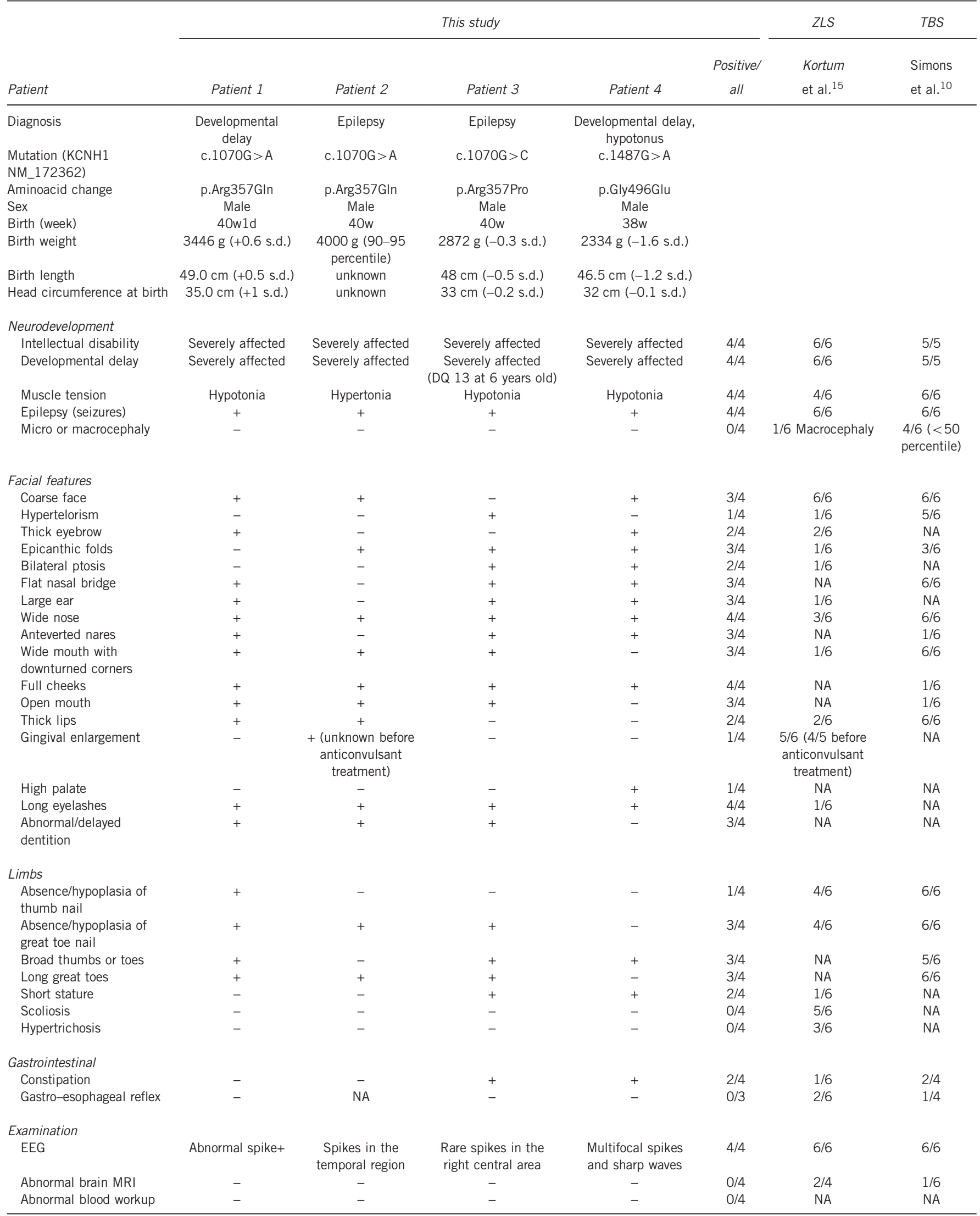

Abbreviations: +, present; -, absent; DQ, developmental quotient; EEG, electroencephalograph; MRI, magnetic resonance imaging; NA, not available; TBS, Temple-Baraitser syndrome; ZLS, Zimmermann-Laband syndrome. 

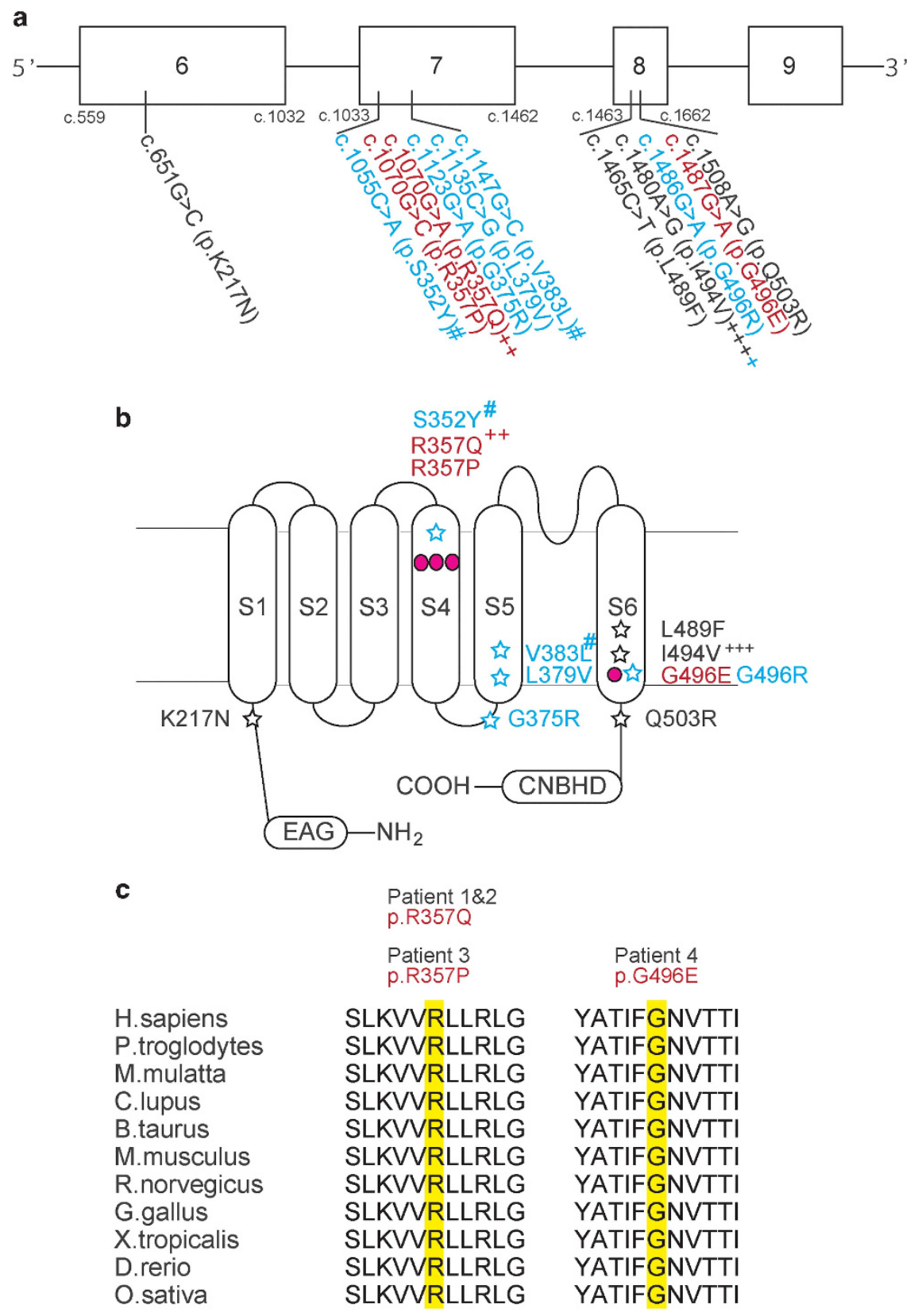

Figure $2 \mathrm{KCNH} 1$ mutations in patients. (a) Genomic structure of exons 6-9 of KCNH1. Colored annotations indicate mutations identified in this study (red), in TBS $^{10}$ (black) and in ZLS patients ${ }^{15}$ (light blue). ++ and ++++ indicate two and four patients had mutations. \# indicates mutations found simultaneously in one patient. (b) Schematic presentation of the KCNH1 (Kv10.1) channel and the location of mutated amino-acid residues found in this (red) and two previous. ${ }^{10,15}$ (black and light blue). (c). The three mutations found in this study occur at evolutionally highly conserved amino acids.

dbSNP137, our in-house 575 control exomes and the Exome Variant Server database. Trio-based WES analysis revealed de novo, autosomal recessive and $\mathrm{X}$-linked recessive candidate variants in two families (Supplementary Table 2). KCNH1 mutations found in four patients were predicted as disease-causing based on by three computational programs sorting Intolerant from Tolerant (SIFT, http://sift.jcvi.org/www/SIFT_enst_submit.html), polymorphism phenotype (PolyPhen-2, http://genetics.bwh.harvard.edu/pph2/), and mutation prediction for the deep-sequencing age (Mutation Taster, http://www.mutationtaster.org/) (Supplementary Table 2). De novo occurrences of all the mutations were confirmed by
Sanger sequencing (Supplementary Figure 1). Locations of the three $\mathrm{KCNH1}$ mutations along with 10 previously reported mutations are illustrated in Figure 2. The c.1070G $>$ A (p.R357Q) was observed in two independent patients and c.1070G $>C$ (p.R357P) was observed in one patient; both were located at an evolutionally highly conserved arginine in the channel voltage-sensor (S4) domain (Figure 2; Table 1). The other mutation, c.1487G $>$ A (p.G496E), was located at an evolutionally highly conserved glycine in the channel pore domain (S6). A similar mutation (p.G496R) at the same position has been reported previously ${ }^{15}$ (Figure 2; Table 1). 


\section{DISCUSSION}

In this study, we identified three novel missense $\mathrm{KCNH} 1$ mutations occurring de novo in four patients with severe developmental delay and infantile epilepsy. As shown in Figure 2a, published data have revealed one ZLS patient with a mutation in exon 6 , three with mutations in exon 7, and five ZLS and two TBS patients with mutations in exon 8. Our series had mutations in exon 7 and 8. Exon 7 mainly encodes domains S4 and S5, whereas exon 8 encodes the S6 domain. Combining the four new patients with the 10 previously reported patients, 15/16 patients had mutations in either exon 7 or 8 , equivalent to domains S4-S6, suggesting these exons are the hotspots for KCNH1-mutant disorders.

The two novel $\mathrm{KCNH} 1$ mutations found in three patients described here are located in the channel voltage-sensor domain (S4). The positively charged residues (arginine and lysine) are responsible for voltage sensing in the S4 domain in Kv channel. ${ }^{2,12,17,18}$ Interestingly, the two detected mutations (p.R357Q and p.R357P) both substituted a positively charged amino-acid residue at Arg357. The repeated arginine motif is important for maintaining the voltage-sensor domain; thus, change from arginine to an uncharged residue (p.R357Q and p.R357P) is highly likely to disrupt the sensitivity and cooperativity of the sensor of the $\mathrm{Kv}$ channel. Although one ZLS patient carried two mutations, one in S4 (p.S352Y) and one in S5 (p.V383L) simultaneously, ${ }^{15}$ the impact of the p.S352Y mutation was not determined. Our reports emphasize the importance of the S4 domain of KCNH1 in humans.

In this report, we have also presented a patient with a mutation, p.G496E, located in the channel pore domain (S6). ${ }^{19}$ This mutation has been previously reported in a patient with ZLS. ${ }^{15}$ Glycine residues in $\mathrm{Kv}$ channels are known to confer flexibility to protein structures ${ }^{20-22}$ so that they act as movable hinges. ${ }^{23}$ Compared with glycine, which is nonpolar and a small amino acid, arginine and glutamate belong to positively and negatively charged groups, respectively, and they are both larger in molecular mass. Therefore, it can be postulated that the molecular size and charge changes of this component of the channel pore would affect the proper functioning of the gate. Mutant KCNH1 (p.G496R) channels expressed in $\mathrm{CHO}$ cells have shown no voltage-dependent $\mathrm{K}$ current, ${ }^{15}$ suggesting that the G496 residue was essential for proper channel gating.

In the four patients described here, the initial symptom was developmental delay, followed by various types of infantile-onset seizures. Some patients have dysmorphic faces, hypotonia and thick broad toes. In addition, three of the four patients had long eyelashes and an open mouth (Figure 1; Table 1). Pathogenic mutations in KCNH1 have been found in ZLS and TBS patients ${ }^{10,15}$ who also display intellectual disability, epilepsy, hypoplasia or aplasia of nails, abnormal muscle tone and craniofacial dysmorphologies (Table 1). It is not easy to clearly differentiate these two syndromes based on clinical phenotypes, and the four patients in this study could not be assigned to either of these syndromes (Table 1). Therefore, we conclude that the mutations in $\mathrm{KCNH} 1$ likely cause a phenotypic continuum of neurodevelopmental disorders covering ZLS and TBS. KCNH1 mutations found in syndromic developmental delay and infantile seizures cluster in S4-S6 domains and highlight the functional importance of those domains in Kv10.1.

In conclusion, we report three de novo missense KCNH1 mutations in four patients with syndromic developmental delay and epilepsy. More information of $\mathrm{KCNH1}$ mutations and their clinical consequences are absolutely needed to delineate the clear phenotype-genotype correlation.

\section{CONFLICT OF INTEREST}

The authors declare no conflict of interest.

\section{ACKNOWLEDGEMENTS}

We thank the patients and their families for participating in this work. This work was supported in part by a grant for Research on Measures for Intractable Diseases, a grant for Comprehensive Research on Disability Health and Welfare, the Strategic Research Program for Brain Science, and a grant for Initiative on Rare and Undiagnosed Diseases in Pediatrics; a Grant-in-Aid for Scientific Research on Innovative Areas (Transcription Cycle) from the Ministry of Education, Culture, Sports, Science, and Technology of Japan; Grants-in-Aid for Scientific Research (A, B and C), and challenging Exploratory Research from the Japan Society for the Promotion of Science; the fund for Creation of Innovation Centers for Advanced Interdisciplinary Research Areas Program in the Project for Developing Innovation Systems from the Japan Science and Technology Agency; the Takeda Science Foundation; the Yokohama Foundation for Advancement of Medical Science; and the Hayashi Memorial Foundation for Female Natural Scientists.

1 Gutman, G. A., Chandy, K. G., Grissmer, S., Lazdunski, M., McKinnon, D., Pardo, L. A. et al. International Union of Pharmacology. LIII. Nomenclature and molecular relationships of voltage-gated potassium channels. Pharmacol. Rev. 57, 473-508 (2005)

2 Miceli, F., Soldovieri, M. V., Ambrosino, P., De Maria, M., Manocchio, L., Medoro, A. et al. Molecular pathophysiology and pharmacology of the voltage-sensing module of neuronal ion channels. Front Cell Neurosci. 9, 259 (2015)

3 Pardo, L. A., del Camino, D., Sánchez, A., Alves, F., Brüggemann, A., Beckh, S. et al. Oncogenic potential of EAG $\mathrm{K}(+)$ channels. EMBO J. 18, 5540-5547 (1999).

4 Haitin, Y., Carlson, A. E. \& Zagotta, W. N. The structural mechanism of KCNH-channel regulation by the eag domain. Nature 501, 444-448 (2013).

5 Biervert, C., Schroeder, B. C., Kubisch, C., Berkovic, S. F., Propping, P., Jentsch, T. J. et al. A potassium channel mutation in neonatal human epilepsy. Science 279, 403-406 (1998).

6 Charlier, C., Singh, N. A., Ryan, S. G., Lewis, T. B., Reus, B. E., Leach, R. J. et al. A pore mutation in a novel KQT-like potassium channel gene in an idiopathic epilepsy family. Nat. Genet. 18, 53-55 (1998).

7 Saitsu, H., Kato, M., Koide, A., Goto, T., Fujita, T., Nishiyama, K. et al. Whole exome sequencing identifies KCNQ2 mutations in Ohtahara syndrome. Ann. Neurol. 72, 298-300 (2012).

8 Weckhuysen, S., Mandelstam, S., Suls, A., Audenaert, D., Deconinck, T., Claes, L. R. et al. KCNQ2 encephalopathy: emerging phenotype of a neonatal epileptic encephalopathy. Ann Neurol. 71, 15-25 (2012).

9 Muona, M., Berkovic, S. F., Dibbens, L. M., Oliver, K. L., Maljevic, S., Bayly, M. A. et al. A recurrent de novo mutation in $\mathrm{KCNC} 1$ causes progressive myoclonus epilepsy. Nat. Genet. 47, 39-46 (2015).

10 Simons, C., Rash, L. D., Crawford, J., Ma, L., Cristofori-Armstrong, B., Miller, D. et al. Mutations in the voltage-gated potassium channel gene $\mathrm{KCNH} 1$ cause Temple-Baraitser syndrome and epilepsy. Nat. Genet. 47, 73-77 (2015).

11 Syrbe, S., Hedrich, U. B., Riesch, E., Djmié, T., Müller, S., Møller, R. S. et al. De novo loss- or gain-of-function mutations in KCNA2 cause epileptic encephalopathy. Nat. Genet. 47, 393-399 (2015).

12 Yang, Y., Vasylyev, D. V., Dib-Hajj, F., Veeramah, K. R., Hammer, M. F., Dib-Hajj, S. D. et al. Multistate structural modeling and voltage-clamp analysis of epilepsy/autism mutation $\mathrm{Kv} 10.2-\mathrm{R} 327 \mathrm{H}$ demonstrate the role of this residue in stabilizing the channel closed state. J. Neurosci. 33, 16586-16593 (2013).

13 Ludwig, J., Terlau, H., Wunder, F., Brüggemann, A., Pardo, L. A., Marquardt, A. et al. Functional expression of a rat homologue of the voltage gated either á go-go potassium channel reveals differences in selectivity and activation kinetics between the Drosophila channel and its mammalian counterpart. EMBO J. 13, 4451-4458 (1994).

14 Gómez-Varela, D., Kohl, T., Schmidt, M., Rubio, M. E., Kawabe, H., Nehring, R. B. et al. Characterization of Eag1 channel lateral mobility in rat hippocampal cultures by single-particle-tracking with quantum dots. PLOS ONE 5, e8858 (2010).

15 Kortüm, F., Caputo, V., Bauer, C. K., Stella, L., Ciolfi, A., Alawi, M. et al. Mutations in $\mathrm{KCNH} 1$ and ATP6V1B2 cause Zimmermann-Laband syndrome. Nat. Genet. 47, 661-667 (2015)

16 Chen, Y., Sánchez, A., Rubio, M. E., Kohl, T., Pardo, L. A. \& Stühmer, W. Functional K (v) 10.1 channels localize to the inner nuclear membrane. PLOS ONE 6, e19257 (2011).

17 Stengel, R., Rivera-Milla, E., Sahoo, N., Ebert, C., Bollig, F., Heinemann, S. H. et al. $\mathrm{Kcnh} 1$ voltage-gated potassium channels are essential for early zebrafish development. J. Biol. Chem. 287, 35565-35575 (2012). 
18 Börjesson, S. I. \& Elinder, F. Structure, function, and modification of the voltage sensor in voltage-gated ion channels. Cell Biochem. Biophys. 52, 149-174 (2008).

19 Wynia-Smith, S. L., Gillian-Daniel, A. L., Satyshur, K. A. \& Robertson, G. A. hERG gating microdomains defined by $\mathrm{S} 6$ mutagenesis and molecular modeling. J. Gen. Physiol. 132, 507-520 (2008).

$20 \mathrm{Li}$, S. C. \& Deber, C. M. Glycine and beta-branched residues support and modulate peptide helicity in membrane environments. FEBS Lett. 311, 217-220 (1992).
21 Beckstein, O., Biggin, P. C., Bond, P., Bright, J. N., Domene, C., Grottesi, A. et al. Ion channel gating: insights via molecular simulations. FEBS Lett. 555, $85-90$ (2003).

22 Bright, J. N. \& Sansom, M. S. P. The flexing/twirling helix: exploring the flexibility about molecular hinges formed by proline and glycine motifs in transmembrane helices. J. Phys. Chem. B 107, 627-636 (2003).

23 Ding, S., Ingleby, L., Ahern, C. A. \& Horn, R. Investigating the putative glycine hinge in Shaker potassium channel. J. Gen. Physiol. 126, 213-226 (2005).

Supplementary Information accompanies the paper on Journal of Human Genetics website (http://www.nature.com/jhg) 